\title{
Asteroseismology of stars on the upper main sequence
}

\section{Patrick Lenz ${ }^{* \dagger}$}

Copernicus Astronomical Center, Polish Academy of Sciences, ul. Bartycka 18, 00-716 Warsaw, Poland

E-mail: lenz@camk.edu.pl

I review the properties of pulsators located on the upper main sequence in the HR diagram and discuss asteroseismic inferences on the internal structure of stars of spectral type A and B. Special attention is given to the problem of uncertainties in stellar opacities in modelling.

Frank N. Bash Symposium New Horizons In Astronomy,

October 9-11, 2011

Austin Texas

\footnotetext{
* Speaker.

${ }^{\dagger}$ The author is partially supported by the Polish MNiSW grant No. N N203 379636.
} 
Pulsating stars on the upper main sequence in the HR diagram commonly exhibit a convective core (which appears if $\mathrm{M}>1.2 \mathrm{M}_{\odot}$ ) and a radiative envelope with thin convection zones close to the surface. The evolutionary status of pulsators located in this region in the HR diagram can be manifold, however: beside common main sequence pulsators (in which hydrogen core burning takes place) we also have pre-main sequence stars (no efficient nuclear reactions) and post-main sequence stars (hydrogen shell burning). We distinguish different types of pulsators along the main sequence band: among $\mathrm{B}$ and late type $\mathrm{O}$ stars we have the so-called $\beta$ Cephei pulsators with periods of hours and masses of 8-20 $\mathrm{M}_{\odot}$ and the long-period SPB oscillators (slowly pulsating B stars) with periods of days and masses of 3-12 $\mathrm{M}_{\odot}$. Moving towards lower masses, there are the $\delta$ Scuti pulsators $\left(\mathrm{M}=1.5-2.5 \mathrm{M}_{\odot}\right)$, which are dwarfs or giants of spectral type A2-F5 located in the extension of the Cepheid instability strip with periods of 0.02-0.3d. Pulsating magnetic stars among A stars are known as roAp pulsators with periods of 5-15 minutes. Among F-type stars there are the $\gamma$ Dor pulsators with masses of 1.4-1.6 $\mathrm{M}_{\odot}$ and periods of $0.3-3 \mathrm{~d}$. Recent observational reviews on these pulsators based on satellite photometry can be found in [1-3] for A and F pulsators and for B stars in $[4,5]$. Prior to the discussion of recent asteroseismic results on the physics in these stars I will review some basics of stellar pulsation.

\section{Stellar oscillations}

Stellar pulsation occurs if a star undergoes free or forced oscillations. In the limit of slow rotation, the star is spherically symmetric in its equilibrium. In this case the geometrical perturbation of the equilibrium through a pulsation mode can be characterized by a spherical harmonic $Y_{\ell}^{m}(\theta, \phi)$, where $\theta$ is the colatitude, $\phi$ the azimuthal angle, $\ell$ the degree (i.e. the number of nodal lines on the surface) and $m$ the number of nodal lines crossing the equator. If $\ell=0$ we have radial pulsation (i.e. a spherically symmetric oscillation) and if $\ell>0$ we have nonradial oscillations. For a spherical degree, $\ell$, the eigenfunctions are degenerate by $(2 \ell+1)$-folds in $m$. Rotation lifts this degeneracy and leads to mode frequencies depending on $m$. Finally, a pulsation mode is also characterized by the number of nodes, $n$, in the radial component of the displacement between the center and the surface. The radial fundamental mode has no node, i.e. $n=0$, the first overtone $n=1$, and so forth.

The symmetry axis of pulsation is commonly aligned to the dominant symmetry axis in the star. It is often the rotation axis; however, in case of the presence of a strong magnetic field it is the magnetic axis, or in a close binary system the tidal axis. If no symmetry axis clearly dominates, the pulsation symmetry axis may lie in between two symmetry axes, e.g., in between the rotation axis and the magnetic field axis [6]. Such an example has already been found in a magnetic A-type star observed by the Kepler satellite [7].

\subsection{Propagation of waves inside a star}

As for every oscillating body its structure and composition determines its frequencies. The solution of the oscillation equations for a star reveals two characteristic (critical) frequencies: (i) the Lamb frequency, $L_{\ell}=\sqrt{\ell(\ell+1)} \frac{c_{s}}{r}$, where $c_{s}$ is the local speed of sound, and (ii) the Brunt-Väisälä frequency, $N$, defined as $N^{2}=g\left(\frac{1}{\Gamma_{1}} \frac{d \ln p_{0}}{d r}-\frac{d \ln \rho_{0}}{d r}\right)$, where the subscript 0 denotes equilibrium values. The Lamb frequency corresponds to the inverse travel time of a sound wave, i.e. a wave front propagates the distance $2 \pi r / \ell$ horizontally within the period $2 \pi / L_{\ell}$. The Brunt-Väisälä frequency 

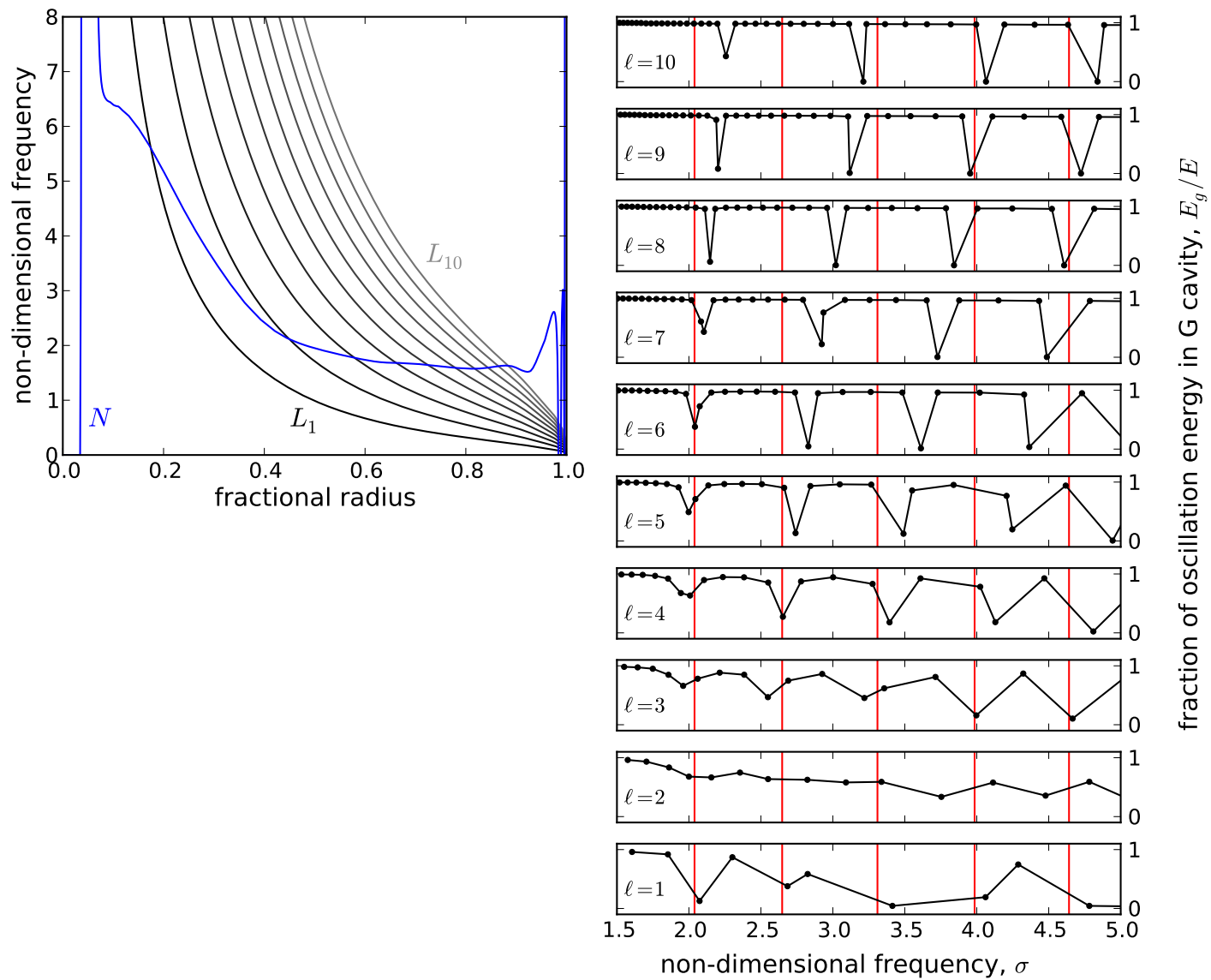

Figure 1: Left panel: propagation diagram for modes up to $\ell=10$ in a model of the $\delta$ Scuti star 44 Tau. Right panel: fraction of oscillation mode energy confined in the $g$ mode propagation zone for modes of different $\ell$. Modes with $E_{g} / E \approx 1$ are effectively trapped in the g-mode cavity, while modes with $E_{g} / E \approx 0$ are trapped in the envelope. The location of the radial modes is indicated by red vertical lines.

describes the frequency of the adiabatic oscillation of a bubble of gas in vertical direction under the influence of buoyancy. Both critical frequencies depend on the local physical conditions inside the star and they determine the cavities in which oscillation may take place. The propagation zone for acoustic waves (p modes) is defined by $\sigma>L_{\ell}$ and $\sigma>N$, where $\sigma$ is the oscillation frequency, and the gravity wave (g mode) propagation zone resides in regions where $\sigma<L_{\ell}$ and $\sigma<N$. In between these two cavities the amplitude of an oscillation mode decreases exponentially with distance, this region is therefore called evanescent zone. Additionally, at high frequencies the acoustic cavity is limited by the acoustic cut-off frequency, above which the oscillation is no longer reflected at the outer boundary but propagates outwards in the atmosphere.

A typical example of propagation zones in a pulsating star of $\approx 1.9 \mathrm{M}_{\odot}$ at the end of hydrogen core burning is given in the left panel of Fig. 1. The high values of $N$ close to the center are due to the strong gradient in mean molecular weight which grows during main sequence evolution because of the receding convective core. This development allows g modes to move to higher frequencies. In the outer envelope two convection zones $(N<0)$ corresponding to partial ionization of $\mathrm{He} \mathrm{II} \mathrm{and}$ 
$\mathrm{HeI} / \mathrm{H}$ are located. A significant part of the energy in these zones is still transported by radiation, but the efficiency of convection increases with decreasing effective temperatures of the star. If the two wave propagation zones are separated only by a small evanescent zone a "tunnel effect" may occur. If the evanescent zone is large enough, however, the propagation zones can be treated as independent and oscillation energy is effectively trapped in a given propagation zone. If this is the case we speak of trapped modes. It can be seen in Fig. 1 that while we expect oscillation modes of higher $\ell$ to be effectively trapped in the envelope or in the interior; for low degree modes only partial trapping occurs. In fact partial trapping is least effective for $\ell=2$ modes in main sequence stars because for these modes the evanescent zone is thinnest in the range of typically excited modes and therefore these modes are strongly coupled to the interior. Consequently, they have both g mode and $\mathrm{p}$ mode properties and are therefore called mixed modes. This effect is also illustrated in the right panel of Fig. 1 which shows the fraction of oscillation energy of a mode confined in the gravity wave propagation zone, $E_{g} / E$. Modes trapped in the envelope, i.e. modes with low $E_{g} / E$, are essentially decoupled from the interior and have the highest probability to be observed. The agglomeration of modes trapped in the envelope close to the frequency of the fundamental radial mode is due to the fact that the modes of all $\ell$ values are limited by the Brunt-Väisälä frequency which itself is $\ell$-independent (see left panel in Fig. 1). Although modes with high spherical degrees suffer from stronger cancellation effects compared to $\ell=1,2$ modes [8] they are now also detectable with present day high-precision satellite photometry.

\section{Excitation and damping of oscillation modes}

What causes an oscillation mode to grow in amplitude? We distinguish between free and forced oscillations. In the latter case a linearly damped oscillation is excited by a periodic external force, e.g., due to a periodic tidal distortion, or generally, due to resonance. In case of free oscillations the oscillations are excited by an internal driving mechanism. Such an excitation mechanism should be located in a region that lies within a propagation zone and the propagation zones should exclude the common damping regions in a star. Moreover, the oscillation mode should not exhibit a node in the driving region. The most relevant mode driving mechanism for self-excited oscillation are outlined in the following paragraphs.

\section{$2.1 \kappa$-mechanism}

Opacity $(\kappa)$, i.e. the quantity which describes the transport of radiation through matter, is temperature-dependent and can act as a valve under certain circumstances. Depending on the layer inside the star, $\kappa$ increases or decreases with increasing temperature which is reflected in the behaviour of the temperature derivative of opacity, $\kappa_{T}$ (see Fig. 2). One condition for the $\kappa$-mechanism to work in a certain region in a star is that this opacity derivative increases in the outward direction. Consequently during a compression phase radiative flux is blocked and performs work as can be seen from the differential work diagram in Fig. 2.

The conditions for pulsational instability of a mode (i.e. for its amplitude to grow with time) was reviewed, e.g., by [9] and only the main points will be repeated here: (i) the amplitude of the pressure eigenfunction has to be large and vary slowly within the driving zone, (ii) the pulsation has to occur faster than the redistribution of thermal energy, i.e. the thermal timescale in the 


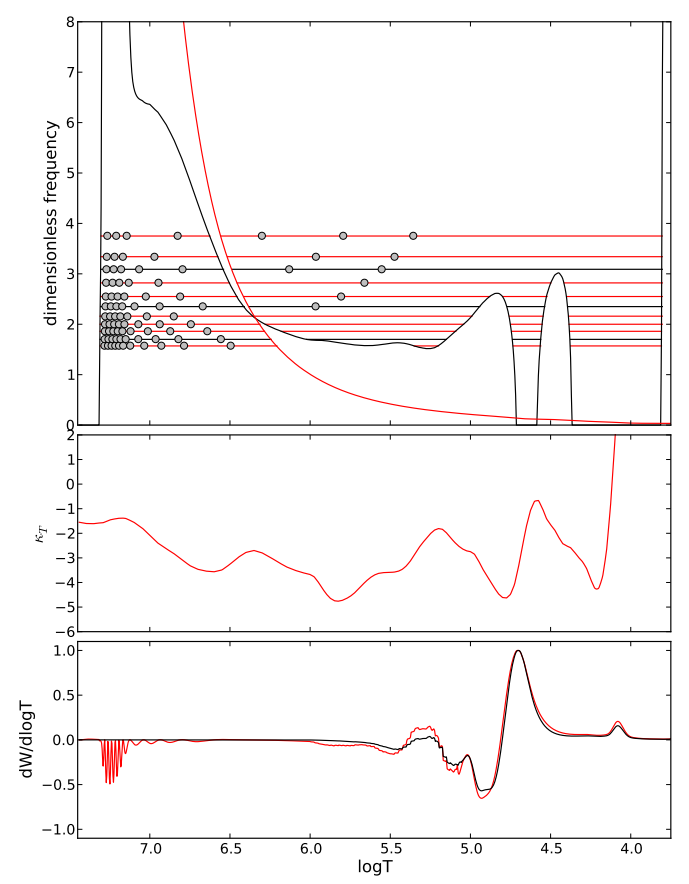

Figure 2: Propagation diagram, temperature derivative of opacity, $\kappa_{T}$, and differential work integral for two $\ell=2$ modes (p mode in black, $g$ mode in red) as a function of $\log T$ in a 44 Tau model.

driving region has to be comparable or longer than the period of the mode. The second condition determines the depth of the driving zone in a star. The given conditions can be met by two frequency ranges in the same star, as for example in hybrid B pulsators: low-order low-degree acoustic or mixed modes with periods of 3-6 hours ( $\beta$ Cep-type pulsation) and high-order low-degree gravity modes with periods of 1.5-3 days (SPB-type pulsation). Condition (ii) implies that these longer period modes are driven in slightly deeper layers than the $\beta$ Cep-type modes.

There are also important damping effects such as radiative dissipation in the gravity mode cavity. The strength of this effect depends on the efficiency of mode trapping in this cavity and it is therefore strong for $g$ modes (see lower panel of Fig. 2). The dissipation increases with the degree of central condensation, which generally increases with age. E.g., Cepheids have a very high central concentration and the strong radiative dissipation in the core region damps g modes before they are reflected at the center. Hence in these stars only acoustic modes trapped in the envelope are observed. In $\delta$ Scuti stars radiative dissipation of modes provides strong damping for g modes of higher $\ell$. At $\ell>12$ only acoustic modes trapped in the envelope remain unstable [8].

\subsection{Convective driving and convective blocking}

Efficient convection may also cause self-excited oscillations. For example in ZZ Ceti stars the convective energy flux in the $\mathrm{H}$-ionization zone is much larger than radiative flux. Since convection occurs on a much shorter timescale compared to g mode oscillations, the instantaneous adjustment of convection to pulsation leads to thermal energy being stored in the convection zone during the compression phase of pulsation (i.e. the convection zone stores heat) thus providing efficient 
driving [10].

However, excitation of $\mathrm{g}$ mode pulsation is also possible if the convective time scale is very long against the pulsation period, such as in F stars in case of $\gamma$ Dor pulsation. The radiative flux may be effectively blocked by convection at the base of the convection zone, which also leads to heating upon compression [11]. For this mechanism to work, the base of the convection zone should match the region where the thermal relaxation time is similar to the pulsation periods of the modes [3].

\subsection{Stochastic driving}

Forced excitation of modes within a certain frequency band due to acoustic noise in an efficient convective envelope is another possible driving mechanism. This excitation mechanism drives the 5-min oscillations in the Sun. Although envelope convection in upper main sequence stars is less efficient than in the Sun, evidence for stochastically excited oscillations has been found in a few stars, partly additional to common opacity mechanism driving. There are examples among A/F stars [12] and O/B stars [13, 14]. However, in one of these cases the stochastic nature of the modes could not be confirmed, since non-linear resonant mode excitation by the large-amplitude radial mode provides similar observational features [15].

\subsection{Modification of mode excitation through a magnetic field}

The presence of a magnetic field modifies driving by the $\kappa$-mechanism in two ways: (i) convection is inhibited in the polar regions of the magnetic field, where inward propagating magnetic slow waves carry away pulsational energy, (ii) more effective gravitational settling leading to hydrogenenriched surface layers. In fact, a nonadiabatic analysis [16] for A-type stars showed that a dipole magnetic field stabilizes low-order acoustic modes at $1 \mathrm{kG}$, while high-order modes of $\ell=1,2$ (roAp-type pulsation) become pulsationally unstable due to driving in the $\mathrm{H}$-ionization zone.

\section{Asteroseismic inferences from upper main sequence stars}

\subsection{Probing stellar opacities}

Opacities are a fundamental ingredient in the calculation of stellar structure and evolution and, therefore, asteroseismic models are very sensitive to them. The opacity coefficients $\kappa\left(T, \rho, X_{i}\right)$ define the interacting cross sections of radiation with matter and determine the efficiency of radiative energy transport in a star. Naturally, these opacity coefficients are high for elements with many electrons. In most of the interior of a main sequence star hydrogen and helium are completely ionized except for the outer envelope. Consequently in the deeper interior heavy elements have a high contribution to the opacity coefficients. Therefore, despite their small mass fractions, heavy elements play an important role in stellar physics.

For practical reasons we commonly use tabulated Rosseland-mean opacities, $\kappa_{R}$, in stellar models. Two sets of opacity tables are currently widely used, the Lawrence Livermore National Laboratory opacity table computed with the OPAL code [17] and the tables of the international Opacity Project collaboration (commonly referred to as OP opacities) [18]. 
In their most recent incarnations the OPAL calculations are based on the 21 most abundant elements in a star while OP considers 17 species (i.e. the same elements as OPAL with exception of $\mathrm{P}, \mathrm{Cl}, \mathrm{K}$ and $\mathrm{Ti}$, which have the lowest abundances in the given mixture). There are also differences in the computational approach, e.g., in the equation of state (EOS) which determines ionization equilibria and level populations required for opacity calculation. The EOS used by OP calculations [19] is based on the 'chemical picture', while the OPAL EOS [20] considers a 'physical picture'. A detailed comparison of both EOS is given in [21].

Seaton et al. [22] compared the Rosseland mean opacities for six elements $(\mathrm{H}, \mathrm{He}, \mathrm{C}, \mathrm{O}, \mathrm{S}$, $\mathrm{Fe}$ ) and generally found good agreement between OPAL and OP. If one compares the OPAL and OP opacities for the full set of species, the differences are more significant, however. The third panel from the top in Fig. 3 shows $\kappa_{\mathrm{OPAL}} / \kappa_{\mathrm{OP}}$ evaluated for the most recent solar element mixture [23] as a function of temperature and density. The most striking difference is the well known fact that the metal opacity bump is shifted to higher temperatures in the OP data. Another feature is that the OPAL table exhibits higher opacities compared to OP at $\log \mathrm{T}=6$ and $\log \left(\rho / T_{6}^{3}\right)=-3$ where $T_{6} \equiv T / 10^{6}$. The figure illustrates the stellar profile of different pulsators such as a $\delta$ Scuti star (1.9 $\left.\mathrm{M}_{\odot}\right)$, a SPB pulsator $\left(4 \mathrm{M}_{\odot}\right)$ and a $\beta$ Cephei star $\left(12 \mathrm{M}_{\odot}\right)$ in the temperature-density plane of the opacity table. As can be seen, different regions in the opacity tables are probed by pulsators on the upper main sequence and the differences between OP and OPAL influence the pulsation models for these stars.

As discussed in the previous section a positive temperature derivative of opacity, $\kappa_{T}$, in outward direction is one important condition for driving pulsations. This condition is fulfilled at the hotter wings of bumps in opacity. We will now discuss the different opacity bumps as shown in the top panel of Fig. 3 and relate them to their corresponding types of pulsators:

The He II bump at $\log \mathrm{T} \approx 4.65$ is due to the second ionization of helium. It is responsible for pulsation in the classical instability strip in the HR diagram, e.g., $\delta$ Scuti pulsators, RR Lyrae stars and Cepheids.

The $\mathbf{Z}$ bump is formed due to a large number of intra-M shell transitions in highly excited ions of iron-group elements which take place at approximately $\log \mathrm{T} \approx 5.3$. Iron has the strongest contribution, but also Nickel is a significant contributor of opacity, despite its lower abundance [24]. The temperature of the Nickel opacity bump is significantly higher in the OP data compared to OPAL which poses an interesting problem. The $\mathrm{Z}$ bump is responsible for pulsational driving in massive main sequence B stars ( $\beta$ Cep and SPB type), but also in evolved stars such as hot subdwarf $\mathrm{B}$ and $\mathrm{O}$ stars ( $\mathrm{sdB}$, sdO) on the extreme horizontal branch. The $\mathrm{Z}$ bump instability strip for radial modes connecting main sequence pulsators of $\beta$ Cep and SPB type with low mass sdO and sdB pulsators is shown, e.g., in Fig. 1 in [25].

The deep opacity bump (DOB) occurs from the partial ionization of L-shell electrons of Iron at $\log \mathrm{T} \approx 6.3$ and of $\mathrm{K}$-shell electrons of $\mathrm{C}, \mathrm{O}, \mathrm{Ne}$ at $\log \mathrm{T} \approx 6.2$. This bump may excite pulsation in hydrogen-rich Wolf-Rayet stars [26] and GW Vir stars [27].

Asteroseismology probes the stellar opacities through different observables for each oscillation mode:

(i) its pulsational instability (in opacity-driven pulsators): as instability critically depends on the opacity profile in the vicinity of the bump responsible for driving (see Fig. 2) [28]

(ii) its frequency: because the oscillation frequencies depend on the radiative properties of the stel- 


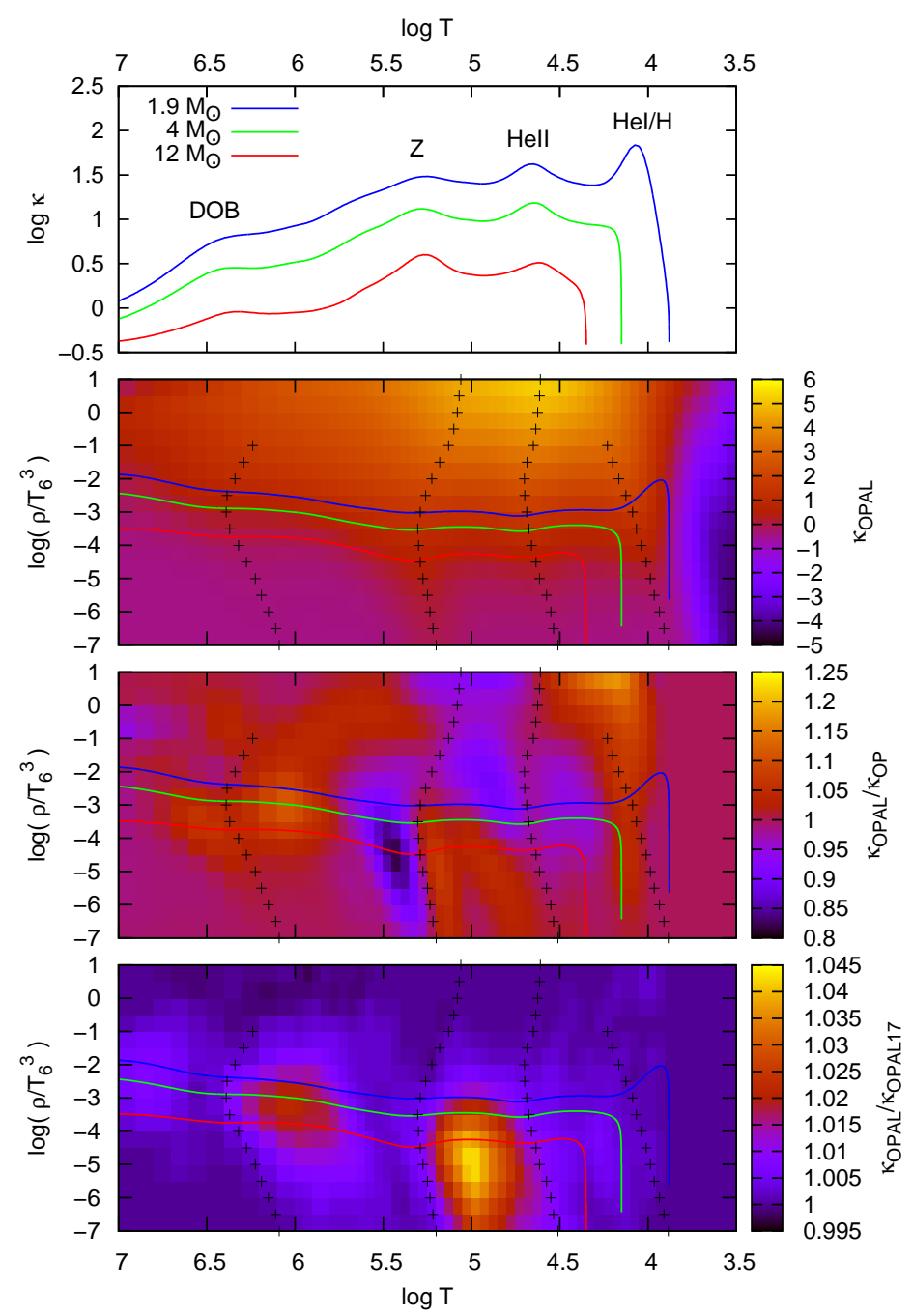

Figure 3: Probing differences in opacity tables by means of main sequence pulsators. The uppermost panel shows Rosseland mean opacity in the envelope of a $\delta$ Scuti model (1.9 $\mathrm{M}_{\odot}$ ), a SPB-type model (4 $\mathrm{M}_{\odot}$ ) and a $\beta$ Cephei model $\left(12 \mathrm{M}_{\odot}\right)$. The adjacent panel shows the profile of these models in the temperaturedensity plane of the OPAL opacity table for $\mathrm{X}=0.74, \mathrm{Z}=0.0134$. Around $\log \mathrm{T}=3.95$ the transition to the low-temperature opacities by [29] occurs. For clarity crosses roughly mark the location of the different opacity bumps. The third panel shows $\kappa_{\mathrm{OPAL}} / \kappa_{\mathrm{OP}}$ in the same plane and the lowermost panel shows the effect of the four elements $(\mathrm{P}, \mathrm{Cl}, \mathrm{K}, \mathrm{Ti})$ which are included in OPAL but not in OP.

lar medium in the propagation zone(s) of the mode.

(iii) its nonadiabatic $f$ parameter (i.e. the ratio of bolometric flux perturbation to the radial displacement at photosphere level): because the complex quantity $f$ probes the nonadiabatic regions and, therefore, is sensitive to the conditions in regions with temperatures below the temperature of the driving opacity bump. Seismic analyses involving the $f$ parameter are commonly termed complex asteroseismology [30, 31].

History has taught us that updates in theoretical opacity calculations (due to improved physics and consideration of additional elements) generally lead to an increase in opacity and an improve- 
ment of our understanding of the excitation of pulsation in stars. For example, based on observational evidence for higher opacity, Simon pleaded for a reinvestigation of heavy element opacities in 1982 [34]. In 1992 the OPAL team published new tables which included new spin-orbit interactions causing an increase of opacity at the $\mathrm{Z}$ bump. This led to the successful explanation of the driving of the observed pulsation in B stars [35, 36]. Consequently, asteroseismology can serve as a tool to help us to identify flaws in certain parts of the opacity tables. Today there is again observational evidence that present day mean opacities underestimate real opacity.

Opacities have been tested by conducting instability surveys (i.e. calculation of instability strips and comparing them to observed positions of pulsators in the HRD) for different types of pulsators. Various effects on the location of the instability strips of upper main sequence pulsators are discussed in [9] and the effect of the latest update of OP opacities and solar element abundances in [37, 38]. Generally, the last update of OP data in 2005 resulted in larger instability domains for both $\beta$ Cephei and SPB pulsators in the HR diagram, and the domains shifted to hotter temperatures. The overlap region that hosts $\beta$ Cephei and SPB hybrid stars is very sensitive to these changes and consequently a good observational probe. For this reason many of these hybrid pulsators were observed in detail during the recent years. Particularly well-studied cases are $12 \mathrm{Lac}$ $\left(\mathrm{M} \approx 11.5 \mathrm{M}_{\odot}\right.$, [39-41]), $v$ Eri $\left(\mathrm{M} \approx 9.5 \mathrm{M}_{\odot},[42-46,40]\right)$ and $\gamma \operatorname{Peg}\left(\mathrm{M} \approx 8.5 \mathrm{M}_{\odot}\right.$, [47-51]). It is striking that in asteroseismic models of these stars certain common problems occur. With OPAL the predicted frequency range of unstable $\beta$ Cephei modes is too narrow. The use of OP data improves the predicted mode instability for $\beta$ Cephei modes in comparison with OPAL but additional instability is needed. With OPAL it is often not possible to excite low-frequency SPB modes at all, while with OP we do obtain unstable high-order g-modes but matching them with observed frequencies is difficult [40]. For example in $\gamma$ Peg $\ell=2$ SPB modes are predicted to be excited with OP opacities but observations indicate $\ell=1$ [50].

The underestimation of driving for $\beta$ Cep modes can be rectified by increasing the opacity bump responsible for driving, since driving is more effective if the bump is more prominent in comparison with its surrounding. An increase in OP opacity by about 50\% at the $\mathrm{Z}$ bump at $\log \mathrm{T} \approx 5.3-5.5$ and an increase of a few $\%$ up to $20 \%$ at the deep opacity bump at $\log \mathrm{T} \approx 6.3$ improves the agreement in terms of excitation and frequency fits for $\beta$ Cephei pulsation [52, 50]. Additional evidence comes from pulsating B stars found in the low-metal environment SMC for which opacity enhancement is needed at the $\mathrm{Z}$ bump to excite the observed modes [53].

Unfortunately there are not many successfully modelled A-F stars. However, there is one star which is well understood and provided some hints on opacities: the $\delta$ Scuti pulsator 44 Tau $\left(\mathrm{M} \approx 1.9 \mathrm{M}_{\odot},[54,55]\right)$. For this star the fact that OP opacities are lower than OPAL by $10 \%$ at $\log \mathrm{T}=6.05$ caused serious problems in modelling, in particular when fitting the period ratio $[56,28]$. This temperature is close to the deep opacity bump and the problem of OP opacities can be solved by an increase of opacity at $\log \mathrm{T}=6.05$ [55] which is close to the temperature region where an opacity increase is also required in $\mathrm{B}$ stars.

Studies focussing on the nonadiabatic $f$ parameter, which is sensitive to opacities in the outer envelope, reveal an ambivalent picture. For the $\beta$ Cep star $\theta$ Ophiuchi $\left(\mathrm{M} \approx 8.2 \mathrm{M}_{\odot}\right.$, [57-60]) as well as for $v$ Eri [62] the comparison between emprically determined $f$ and theoretically computed values show preference for OPAL opacities [61, 62]. In $\gamma$ Peg, however, models based on both OP and OPAL fail to reproduce the nonadiabatic properties of observed SPB-type modes [63]. 
Consequently, [40] pointed out the requirement of enhancement of opacity in the driving region in order to explain the instability of SPB-type frequencies in these hybrid pulsators. In B stars opacity enhancement tests have the following effect: increasing opacity at the driving bump (e.g., the Z bump in B stars) affects mode instability for SPB and $\beta$ Cep pulsation but does not change the frequencies significantly because the opacity was changed at low densities. Since the DOB is located in denser layers it influences the mode frequencies strongly and hence, if we fit theoretical frequencies to observed counterparts, the position in the HR diagram changes. Additional evidence for more opacity also comes from recent observations hardening the claim of $\beta$ Cep-type pulsations in O stars, e.g., the O9V star HD 46202 [64], or the O9.5V star $\zeta$ Oph [72]. These observations could be explained by widening the $\beta$ Cep instability strip by means of additional opacity at the $\mathrm{Z}$ bump. In the Sun an opacity enhancement of $30 \%$ at the base of the convective zone (which approximately corresponds to the deep opacity bump) and a few percent in the solar core has also been suggested to solve the discrepancy between the solar model and helioseismology based on the current version of solar abundances [65].

A possible explanation for underestimated opacity, apart from uncertainties in the calculations (especially concerning the peak temperature of the Nickel bump), is the possible opacity contribution from elements with low abundances which currently are not included in opacity calculations. In the OPAL calculations four species $(\mathrm{P}, \mathrm{Cl}, \mathrm{K}, \mathrm{Ti})$ are considered which are not included in the OP computations. Using the OPAL web interface ${ }^{1}$ we retrieved a table which adopts the element abundances from the most recent solar mixture [23] but setting the number fraction of these four elements to zero. Since this table is then based only on 17 elements we denote this table as OPAL17 hereafter. Due to the renormalization of the number fractions, we have a minor abundance increase in all metal elements. The lowest panel in Fig. 3 shows the ratio $\kappa_{\mathrm{OPAL}} / \kappa_{\mathrm{OPAL} 17}$ for the solar chemical composition illustrating where these four elements contribute opacity. They augment up to a few $\%$ of opacity for upper main-sequence stars at the hot wing of the $\mathrm{Z}$ bump around $\log \mathrm{T} \approx 5$ and close to the $\mathrm{DOB}$ at $\log \mathrm{T} \approx 6.0$. Consequently one may conclude that the inclusion of more species may partly solve the problems in asteroseismic modelling of star on the upper main sequence. We also note that for denser stars with masses comparable to the Sun the contribution is negligible which confirms the findings of [66].

The differences between OP and OPAL concerning the opacity peak temperature of Nickel showed that there are interesting things to be learned from the opacity calculations. The need for a reinvestigation was also realized by atomic physicists. Therefore, new activities in the determination of opacity for astrophysical purposes on both the theoretical and experimental side have started [67]. The theoretical activities include for example the comparison of spectral opacities for certain elements between different theoretical groups. It is important to validate the theoretical results with experimentally determined spectral opacities using modern high-energy laser facilities. These tests are important to check whether the calculations use proper physics. While the stellar densities are too low to be reproduced in the laboratory, it is possible to draw conclusions by studying equivalent plasma conditions that have similar mean ionization states. New calculations concentrate on the conditions at the base of the solar convection zone and in the driving zone in B stars. Preliminary results of a comparison of calculated spectral opacities (with 8 codes participating) and experiments

\footnotetext{
${ }^{1}$ http://opalopacity.llnl.gov/new.html
} 
done at the LULI 2000 facility in France are given in [67-69]. These experiments/calculations are not only important to determine accurate mean opacities; in fact accurate spectral opacities are very important to determine reliable opacity coefficients for radiative accelerations.

\subsection{Chemical evolution}

Stellar opacities depend on the chemical composition. For example there is observational evidence that the photospheric abundances of certain metal species of B stars may be lower than solar [32] and it was shown [33] that this chemical composition leads to a higher opacity peak at $\log \mathrm{T}=5.3$ and produces a wider instability strip. In computations one often assumes the chemical abundances to be homogeneous in the whole envelope. However, atomic diffusion, unless hampered by mixing effects, rearranges elements.

Atomic diffusion, see e.g. [70], is a slow process that modifies the local element abundances due to the counterplay between radiative acceleration vs. gravitional settling which is different for ions of different species. Regrettably, there are currently large uncertainties in the determination of radiative accelerations since they depend on spectral opacities. In main sequence stars atomic diffusion is responsible for shaping the superficial abundance pattern of Ap and Cp stars. Diffusion may also be partly responsible for the enhancement of opacity around the $\mathrm{Z}$ bump in $\mathrm{B}$ stars, because elements accumulate due to diffusion where their specific opacity is large. The effect of diffusion is however swept out if mixing processes are effective.

Element mixing in main sequence stars occurs due to different processes such as convection, convective core overshooting and rotationally induced element mixing like meridional circulation. These processes smooth the stratification of elements and our knowledge about their efficiency is still subject to uncertainties.

Among these processes, the extent of overshooting above the convective core is the easiest one to be measured observationally. Common values for slowly rotating $\beta$ Cep stars are an overshooting layer with an extent of $0.1-0.4$ pressure scale heights [73]. To disentangle the effect of overshooting from rotationally induced mixing, studies of more rapid rotators are needed [40]. Asteroseismic analyses of rapid rotators, however, require 2D models which are currently in development [74]. Nonetheless, the derived overshooting parameter also depends on the chemical stratification and the corresponding opacities.

\section{Outlook on the near future}

Solving the remaining problems related to mean opacities is important to obtain accurate asteroseismic models. Precisely determined stellar masses and other fundamental parameters are also important in studies on exoplanets [75]. Satellite missions devoted to the detection of earth-like planets such as Kepler and CoRoT are currently continuing their observations and provide excellent data for asteroseismic studies. The Canadian mission MOST is also still delivering data despite exceding its projected mission lifetime.

New projects are on the horizon:

BRITE constellation ${ }^{2}$ is an Austrian-Polish-Canadian mission consisting of a set of 6 nanosatellites to observe luminosity variations of bright stars. The unique feature of this mission is that

\footnotetext{
${ }^{2}$ http://www.brite-constellation.at
} 
the satellites are equipped with a filter in a red or blue passband respectively. The amplitude and phase difference between the two wavelength bands allows for the photometric determination of the surface geometry of pulsation modes at an unprecedent accuracy for bright stars. The launch of the first pair of satellites is scheduled for spring 2012.

The SONG project (short for: Stellar Observations Network Group) consists of a network of 1-meter robotic telescopes devoted to observing bright stars to do asteroseismology and followup observations of exoplanet hosts. Each node of this network is equipped with a high-resolution echelle spectrograph. The prototype node in Tenerife is expected to deliver first light by the end of $2011[71]$.

Along with additional observations theoretical models and asteroseismic tools are being improved. One example is the open access evolutionary code within the MESA package [76] which is rapidly developed and currently adapted to asteroseismic use.

Consequently the future of asteroseismology is bright.

\section{References}

[1] Uytterhoeven, K., Moya, A., Grigahcéne, A., et al., The Kepler characterization of the variability among A- and F-type stars, 2011, A\&A, 534, A125

[2] Balona, L. A., \& Dziembowski, W. A., Kepler observations of $\delta$ Scuti stars, 2011, MNRAS, 417, 591

[3] Balona, L. A., Guzik, J. A., Uytterhoeven, K., et al., The Kepler view of $\gamma$ Doradus stars, 2011, MNRAS, 415, 3531

[4] Balona, L. A., Pigulski, A., De Cat, P., et al., Kepler observations of the variability in B-type stars, 2011, MNRAS, 413, 2403

[5] Degroote, P., Aerts, C., \& Ollivier, M., CoRoT's view of newly discovered B-star pulsators: results for 358 candidate $B$ pulsators from the initial run's exoplanet field data, 2009, A\&A, 506, 471

[6] Bigot, L., \& Dziembowski, W. A., The oblique pulsator model revisited, 2002, A\&A, 391, 235

[7] Kurtz, D. W., Cunha, M. S., Saio, H., et al., The first evidence for multiple pulsation axes: a new rapidly oscillating Ap star in the Kepler field, KIC 10195926, 2011, MNRAS, 414, 2550

[8] Balona, L. A. \& Dziembowski, W. A., Excitation and visibility of high-degree modes in stars, 1999, MNRAS, 309, 221

[9] Pamyatnykh, A. A., Pulsational Instability Domains in the Upper Main Sequence, 1999, AcA, 49, 119

[10] Goldreich, P., \& Wu, Y., Gravity Modes in ZZ Ceti Stars. I. Quasi-adiabatic Analysis of Overstability, 1999, ApJ, 511, 904

[11] Guzik, J. A., Kaye, A. B., Bradley, P. A., et al., Driving the Gravity-Mode Pulsations in $\gamma$ Doradus Variables, 2000, ApJ, 542, 57

[12] Antoci, V., Handler, G., Campante, T. L., et al., The excitation of solar-like oscillations in a $\delta$ Sct star by efficient envelope convection, 2011, Nature, 477, 570

[13] Belkacem, K., Samadi, R., Goupil, M.-J., et al., Solar-Like Oscillations in a Massive Star, 2009, Science, 324, 1540

[14] Degroote, P., Briquet, M., Auvergne, M., et al., Detection of frequency spacings in the young O-type binary HD 46149 from CoRoT photometry, 2010, A\&A, 519, 38 
[15] Aerts, C., Briquet, M., Degroote, P., et al., Seismic modelling of the $\beta$ Cephei star HD 180642 (V1449 Aquilae), 2011, A\&A, 534, 98

[16] Saio, H., A non-adiabatic analysis for axisymmetric pulsations of magnetic stars, 2005, MNRAS, 360, 1022

[17] Iglesias, C. A., \& Rogers, F. J., Updated Opal Opacities, 1996, AJ, 464, 943

[18] Badnell, N. R., Bautista, M. A., Butler, K., et al., Updated opacities from the Opacity Project, 2005, MNRAS, 360, 458

[19] Hummer, D. G., \& Mihalas, D., The equation of state for stellar envelopes. I , 1988, ApJ, 331, 794

[20] Rogers, F. J., \& Nayfonov, A., Updated and Expanded OPAL Equation-of-State Tables: Implications for Helioseismology, 2002, ApJ, 576, 1064

[21] Trampedach, R., Däppen, W., \& Baturin, V. A., A Synoptic Comparison of the Mihalas-Hummer-D̈̈pen and OPAL Equations of State, 2006, ApJ, 646, 560

[22] Seaton, M. J., \& Badnell, N. R., A comparison of Rosseland-mean opacities from OP and OPAL, 2004, MNRAS, 354, 457

[23] Asplund, M., Grevesse, N., Sauval, J. A., \& Scott, P., The Chemical Composition of the Sun, 2009, Annual Review of Astronomy \& Astrophysics, 47, Issue 1, 481

[24] Jeffery, C. S., \& Saio, H., Gravity-mode pulsations in subdwarf B stars: a critical test of stellar opacity, 2006, MNRAS, 372, 48

[25] Jeffery, C. S., \& Saio, H., Improved Opacities and Pulsation Models for sdB and sdO Stars, 2008, ASPC , 392, 245

[26] Townsend, R. H. D., \& MacDonald, J., Excitation of g modes in Wolf-Rayet stars by a deep opacity bump, 2006, MNRAS, 368, 57

[27] Gautschy, A., Althaus, L. G., \& Saio, H., On the excitation of PG 1159-type pulsations, 2005, A\&A, 438,1013

[28] Montalbán, J., \& Miglio, A., Input from opacity data in computation of pulsation instability, 2008, CoAst, 157, 160

[29] Ferguson, J. W., Alexander, D. R., Allard, F., et al., Low-Temperature Opacities, 2005, ApJ, 623, 585

[30] Daszyńska-Daszkiewicz, J., \& Walczak, P., Complex asteroseismology of the B-type main sequence pulsators, 2010, Highlights of Astronomy, 15, 364

[31] Daszyńska-Daszkiewicz, J., \& Walczak, P., Constraints on opacities from complex asteroseismology of B-type pulsators: the $\beta$ Cephei star $\theta$ Ophiuchi, 2009, MNRAS, 398, 1961

[32] Morel, T., Abundances of massive stars: some recent developments, 2009, CoAst, 158, 122

[33] Montalbán, J., Miglio, A., \& Morel, T., Uncertainties in the chemical composition of B-type stars: effects on the opacity and on the excitation of pulsation modes, 2009, CoAst, 158, 288

[34] Simon, N. R., A plea for reexamining heavy element opacities in stars, 1982, ApJ, 260, 87

[35] Dziembowski, W. A., \& Pamiatnykh, A. A., The opacity mechanism in B-type stars. I - Unstable modes in Beta Cephei star models, 1993, MNRAS, 262, 204

[36] Dziembowski, W. A., Moskalik, P., \& Pamyatnykh, A. A., The Opacity Mechanism in B-Type Stars Part Two - Excitation of High-Order G-Modes in Main Sequence Stars, 1993, MNRAS, 265, 588 
[37] Miglio, A., Montalbán, J., \& Dupret, M.-A., Instability strips of slowly pulsating B stars and $\beta$ Cephei stars: the effect of the updated OP opacities and of the metal mixture, 2007, MNRAS, 375, 21

[38] Zdravkov, T., \& Pamyatnykh, A. A., Main-sequence instability strip for different opacities and heavy element abundances: a comparison with observations, 2008, Jour. of Phys.: Conf. Series, 118, 012079

[39] Handler, G., Jerzykiewicz, M., Rodríguez, E., et al., Asteroseismology of the $\beta$ Cephei star 12 Lac: photom. observations, pulsational frequency analysis \& mode identification, 2006, MNRAS, 365, 327

[40] Dziembowski, W. A. and Pamyatnykh, A. A., The two hybrid B-type pulsators: v Eridani and 12 Lacertae, 2008, MNRAS, 385, 2061

[41] Desmet, M., Briquet, M., Thoul, A., et al., An asteroseismic study of the $\beta$ Cephei star 12 Lac: multisite spectrosc. observations, mode identification \& seismic modelling, 2009, MNRAS, 396, 1460

[42] Handler, G., Shobbrook, R. R., Jerzykiewicz, M., et al., Asteroseismology of the $\beta$ Cephei star v Eridani - I. Photometric observations and pulsational frequency analysis, 2004, MNRAS, 347, 454

[43] Aerts, C., De Cat, P., Handler, G., et al., Asteroseismology of the $\beta$ Cephei star v Eridani - II. Spectroscopic observations and pulsational frequency analysis, 2004, MNRAS, 347, 463

[44] De Ridder, J., Telting, J. H., Balona, L. A., et al., Asteroseismology of the $\beta$ Cephei star $v$ Eridani III. Extended frequency analysis and mode identification, 2004, MNRAS, 351, 324

[45] Pamyatnykh, A. A., Handler, G., \& Dziembowski, W. A., Asteroseismology of the $\beta$ Cephei star v Eridani: interpretation and applications of the oscillation spectrum, 2004, MNRAS, 350, 1022

[46] Jerzykiewicz, M., Handler, G., Shobbrook, R. R., et al., Asteroseismology of the $\beta$ Cephei star v Eridani - IV. The 2003-2004 multisite photometric campaign and the combined 2002-2004 data, 2005, MNRAS, 360, 619

[47] Chapellier, E., Le Contel, D., Le Contel, J. M., et al., A hybrid $\beta$ Cephei-SPB star in a binary system: $\gamma$ Pegasi, 2006, A\&A, 448, 697

[48] Handler, G., Matthews, J. M., Eaton, J. A., et al., Asteroseismology of Hybrid Pulsators Made Possible: Simultaneous MOST Space Photometry and Ground-Based Spectroscopy of $\gamma$ Peg, 2009, ApJ, 698, 56

[49] Handler, G., Confirmation of simultaneous $p$ and $g$ mode excitation in HD 8801 and $\gamma$ Peg from time-resolved multicolour photometry of six candidate 'hybrid' pulsators, 2009, MNRAS, 398, 1339

[50] Zdravkov, T., \& Pamyatnykh, A. A., Modelling hybrid $\beta$ Cephei/SPB pulsations: $\gamma$ Pegasi, 2009, AIPC, 1170, 388

[51] Pandey, C. P., Morel, T., Briquet, M., et al., A spectroscopic study of the hybrid pulsator Gamma Pegasi, 2011, CoAst, 162, 21

[52] Zdravkov, T., \& Pamyatnykh, A. A., Can opacity changes help to reproduce the hybrid star pulsations?, 2008, CoAst, 157, 385

[53] Salmon, S., Montalbán, J., Miglio, A., et al., Can an underestimation of opacity explain B-type pulsators in the SMC?, 2010, arXiv:1007.3088

[54] Antoci, V., Breger, M., Rodler, F., et al., Is 44 Tauri an exceptional case among the $\delta$ Scuti stars?, 2007, $A \& A, 463,225$

[55] Lenz, P., Pamyatnykh, A. A., Zdravkov, T., \& Breger, M., A $\delta$ Scuti star in the post-MS contraction phase: 44 Tauri, 2010, A\&A, 509, 90 
[56] Lenz, P., Pamyatnykh, A. A., \& Breger, M., The Effect of Different Opacity Data and Chemical Element Mixture on the Petersen Diagram, 2007, AIPC, 948, 201

[57] Handler, G., Shobbrook, R. R., \& Mokgwetsi, T., An asteroseismic study of the $\beta$ Cephei star $\theta$ Ophiuchi: photometric results, 2005, MNRAS, 362, 612

[58] Briquet, M., Lefever, K., Uytterhoeven, K., \& Aerts, C., An asteroseismic study of the $\beta$ Cephei star $\theta$ Ophiuchi: spectroscopic results, 2005, MNRAS, 362, 619

[59] Briquet, M., Morel, T., Thoul, A., et al., An asteroseismic study of the $\beta$ Cephei star $\theta$ Ophiuchi: constraints on global stellar parameters and core overshooting, 2007, MNRAS, 381, 1482

[60] Lovekin, C. C., Goupil, M.-J., Rotation and convective core overshoot in $\theta$ Oph, 2010, A\&A, 515, 58

[61] Daszyńska-Daszkiewicz, J., \& Walczak, P., Constraints on opacities from complex asteroseismology of B-type pulsators: the $\beta$ Cephei star $\theta$ Ophiuchi, 2009, MNRAS, 398, 1961

[62] Daszyńska-Daszkiewicz, J., \& Walczak, P., A complex asteroseismic study of the hybrid B-type pulsator $v$ Eridani, 2010, Ap\&SS, 328, 97

[63] Walczak, P., \& Daszyńska-Daszkiewicz, J., Complex asteroseismology of the hybrid B-type pulsator $\gamma$ Pegasi: A test of stellar opacities, 2010, AN, 331, 1057

[64] Briquet, M., Aerts, C., Baglin, A., et al., An asteroseismic study of the O9V star HD 46202 from CoRoT space-based photometry, 2011, A\&A, 527, 112

[65] Christensen-Dalsgaard, J., di Mauro, M. P., Houdek, G., \& Pijpers, F., On the opacity change required to compensate for the revised solar composition, 2009, A\&A, 494, 205

[66] Guzik, J. A., Keady, J. J., \& Kilcrease, D. P., Early Solar Mass Loss, Opacity Uncertainties, and the Solar Abundance Problem, 2009, AIPC, 1170, 577

[67] Turck-Chièze, S., Loisel, G., Gilles, D., et al., Theoretical and experimental activities on opacities for a good interpretation of seismic stellar probes, 2011, Jour. of Phys.: Conf. Series, 271, 012035

[68] Turck-Chièze, S., Loisel, G., Gilles, D., et al., Radiative properties of stellar plasmas and open challenges, 2011, Ap\&SS, 336, 103

[69] Gilles, D., Turck-Chièze, S., Loisel, G., et al., Comparison of Fe and Ni opacity calculations for a better understanding of pulsating stellar envelopes, 2011, HEDP, 7, 312

[70] Alecian, G., Gravitational settling and radiative accelerations, 2009, CoAst, 158, 34

[71] Creevey, O. L., Grundahl, F., Pallé, P. L., et al., The Observatorio del Teide welcomes SONG:the Stellar Observations Network Group, 2012, Highlights of Spanish Astrophysics VI, Proceedings of the IX Scientific Meeting of the Spanish Astronomical Society (SEA), 686

[72] Walker, G. A. H., Kuschnig, R., Matthews, J. M., et al., Pulsations of the Oe Star $\zeta$ Ophiuchi from MOST Satellite Photometry and Ground-based Spectroscopy, 2005, ApJ, 623, 145

[73] Thoul, A., Asteroseismology of B stars, 2009, CoAst, 159, 35

[74] Reese, D. R., Oscillations in rapidly rotating stars, 2010, AN, 331, 1038

[75] Moya, A., Pulsating stars harbouring planets, 2012, arXiv:1111.2274

[76] Paxton, B., Bildsten, L., Dotter, A., et al., Modules for Experiments in Stellar Astrophysics (MESA), 2011, ApJS, 192, 3 